\title{
LOCAL COHOMOLOGY OF REES ALGEBRAS AND HILBERT FUNCTIONS
}

\author{
BERNARD JOHNSTON AND JUGAL VERMA
}

(Communicated by Wolmer Vasconcelos)

\begin{abstract}
Let $I$ be an ideal primary to the maximal ideal in a local ring. We utilize two well-known theorems due to J.-P. Serre to prove that the difference between the Hilbert function and the Hilbert polynomial of $I$ is the alternating sum of the graded pieces of the graded local cohomology (with respect to its positively-graded ideal) of the Rees ring of $I$. This gives new insight into the higher Hilbert coefficients of $I$. The result is inspired by one due to J. D. Sally in dimension two and is implicit in a paper by D. Kirby and H. A. Mehran, where very different methods are used.
\end{abstract}

\section{INTRODUCTION}

Throughout this note, let $(A, \mathfrak{M}, d)$ be a local ring, by which we mean Noetherian and commutative having Krull dimension $d$, and let $I$ be an $\mathfrak{M}$ primary ideal of $A$. Classically, one has sought knowledge about the ideal $I$ through the study of the Hilbert polynomial of $I$, i.e., the unique polynomial in $n$ whose value is precisely the length of $A / I^{n}$ (as an $A$-module) for all large $n$. The (normalized) leading coefficient of the Hilbert polynomial, the multiplicity of the ideal $I$, is relatively well understood, but the other coefficients remain to this day something of a mystery. Recently, progress in the study of these other Hilbert coefficients has been made by several authors (cf. [G], [HJLS1], [HJLS2], [HJL], [Hn], [Ma], [Mo], [O], [Sa]).

The purpose of this paper is to use two well-known theorems of J.-P. Serre's in order to give a short and sweet proof of a striking relationship between, on the one hand, the coefficients of the Hilbert polynomial of $I$ and, on the other hand, the lengths of various pieces of the graded local cohomology modules (with respect to its positively graded ideal) of the Rees algebra of $A$ with respect to $I$ (see $\S 1$ below for an explanation of terminology and notation). Namely, we prove the following theorem:

Received by the editors February 17, 1993.

1991 Mathematics Subject Classification. Primary 13A30, 13D40, 13 D45.

Key words and phrases. Hilbert polynomial, local cohomology, Rees ring.

The first author was partially supported by a grant from the National Science Foundation. 
Theorem 2.4. If $(A, \mathfrak{M}, d)$ is a local ring and if $I$ is an $\mathfrak{M}$-primary ideal of $A$ then for all $n \geq 0$,

$$
\sum_{i=0}^{d}(-1)^{i} \ell_{A}\left(H_{R_{+}}^{i}(R)_{n}\right)=(-1)^{d}\left[P_{I}(n)-H_{I}(n)\right] .
$$

Here, $R$ denotes the Rees algebra of $A$ with respect to $I$, and $R_{+}$its positively graded ideal.

This result simplifies and clarifies several results in the literature; in particular, the main result of [K-M], Theorem 2, and the following proposition due to J. D. Sally, which was the beginning of our study, in the case of nonnegative $n$.

Proposition [Sa, Proposition 5]. Let $(A, \mathfrak{M}, 2)$ be a Cohen-Macaulay local ring, and write the Hilbert polynomial of $A$ with respect to $\mathfrak{M}$ as $P_{\mathfrak{M}}(n)=$ $e_{0}\left(\begin{array}{c}n+1 \\ 2\end{array}\right)-e_{1}\left(\begin{array}{l}n \\ 1\end{array}\right)+e_{2}$. Then there exists a relation between the graded pieces of the second local cohomology of the Rees algebra with respect to its positively graded ideal and the Hilbert coefficients, as follows:

$$
\begin{aligned}
\ell_{A}\left(H_{R_{+}}^{2}(R)_{0}\right) & =e_{2}, \\
\ell_{A}\left(H_{R_{+}}^{2}(R)_{1}\right) & =e_{0}-e_{1}+e_{2}-1, \\
\ell_{A}\left(H_{R_{+}}^{2}(R)_{-1}\right) & =e_{1}+e_{2} .
\end{aligned}
$$

After a few preliminaries in $\S 1$, we proceed to the proof of our main result in $\S 2$, and then we conclude the paper by examining some special cases in $\S 3$. In particular, we will see in the latter section how our result is a considerable generalization of Sally's Proposition 5.

The approach taken by D. Kirby and H. A. Mehran in [K-M] does not use graded local cohomology at all, using instead the study of a certain subcomplex of a Koszul complex on certain powers of the generators of the ideal $I$. The use of graded local cohomology, besides being simpler, has the advantage that it avoids altogether the difficulty of proving that the result obtained is independent of the powers of the elements chosen, [K-M, Theorem 5]. The chief difficulty in either approach appears to be proving the finiteness of the length of certain modules which appear in the calculations. This is accomplished in the cited paper by an inductive argument based on a certain quotient complex, whereas, in our approach, we reduce the problem to a geometric argument, so the finiteness is seen to follow directly from Serre's Theorem on the finiteness of sheaf cohomology modules, Theorem 2.3 below.

It is a pleasure to thank our erstwhile colleague, A. Nobile, for some helpful conversations during the preparation of this paper.

\section{Preliminaries}

We begin with a few remarks on notation. For a ring $A$ having ideal $I$, we shall denote the Rees algebra of $A$ with respect to $I$ (i.e., the ring $A \oplus I \oplus I^{2} \oplus$ $\ldots \cong A[I t], t$ an indeterminate) with the notation $R$, and we shall denote the associated graded ring of $A$ with respect to $I$ (i.e., the ring $A / I \oplus I / I^{2} \oplus \cdots$ ) with the notation $G . \ell_{A}(M)$ will always denote the length over $A$ of the $A$ module $M$.

$H_{I}(n):=\ell_{A}\left(A / I^{n}\right)$ will always denote the Hilbert function of $R$ with respect to $I$ and $P_{I}(n)$ the corresponding Hilbert polyromial. There exists con- 
siderable disagreement in the literature as to the proper way to normalize the coefficients of the Hilbert polynomial (all agree on the leading coefficient). In this paper we shall adopt the convention which seems to be the most standard in recent years, namely:

$$
P_{I}(n)=e_{0}\left(\begin{array}{c}
n+d-1 \\
d
\end{array}\right)-e_{1}\left(\begin{array}{c}
n+d-2 \\
d-1
\end{array}\right)+\cdots+(-1)^{d} e_{d} .
$$

We will assume throughout that $A$ has an infinite residue field: we need to know that an $\mathfrak{M}$-primary ideal in $A$ has a minimal reduction consisting of $d$ elements. All homomorphisms between graded modules over a graded ring are assumed to be of degree zero. A good reference for information about the category of graded modules over a graded ring is the book [HIO].

Let us briefly recall the notion of local cohomology in the graded context. Let $B$ be a Noetherian $\mathbb{Z}$-graded ring having a homogeneous ideal $J$ and let $M$ be a $\mathbb{Z}$-graded $B$-module. Let $a_{1}, \ldots, a_{r}$ be a set of homogeneous elements of $B$ so that $\left(a_{1}, \ldots, a_{r}\right)$ has the same radical as $J$. Then the $i$ th local cohomology is the $i$ th cohomology of the "Čech" (co-)complex $C \cdot\left(a_{1}, \ldots, a_{r} ; M\right)$, whose $p$ th module is simply the direct sum $\bigoplus_{1 \leq i_{1}<\cdots<i_{p} \leq r} M_{a_{i_{1}} \cdots a_{i_{p}}}$ of all localizations of $M$ using the $a_{i}$ 's, taken $p$ at a time.

The boundary maps of this complex are derived from the canonical localization maps by taking alternating sums in such a manner that this complex is precisely the direct limit of the successive Koszul cocomplexes on $M$, obtained by taking successively higher powers of the individual $a_{i}$ 's; as the definition of these maps is straightforward and a bit technical, we refer the reader to [ibid., top of p. 304] for details. We emphasize here that the modules of the form $M_{a_{i_{1}} \cdots a_{i_{p}}}$ inherit a $\mathbb{Z}$-grading from that of $M$, and hence so do the components of $C \cdot\left(a_{1}, \ldots, a_{r} ; M\right)$. Moreover, as the boundary maps turn out to be graded, the local cohomology modules thereby have a natural grading. In fact, even a more general grading on $M$ will likewise be inherited by the local cohomology modules. Several other equivalent formulations of the graded local cohomology modules are given in $\S 35$ of [ibid.].

It is quite useful in our context to recall the two exact sequences which connect local and global (sheaf) cohomology, namely,

$$
0 \rightarrow H_{R_{+}}^{0}(R) \rightarrow R \rightarrow \bigoplus_{n \in \mathbb{Z}} H^{0}\left(X, \mathscr{O}_{X}(n)\right) \rightarrow H_{R_{+}}^{1}(R) \rightarrow 0
$$

and

$$
\bigoplus_{n \in \mathbb{Z}} H^{i}\left(X, \mathscr{O}_{X}(n)\right) \cong H_{R_{+}}^{i+1}(R), \quad i \geq 1,
$$

where $X:=$ Proj $R$.

It is necessary for us to compare the local cohomology of $R$ with that of $G$. This is accomplished in Lemma 1.2 below. We first need a more technical lemma.

Lemma 1.1. Let $(A, \mathfrak{M}, d)$ be a local ring with $I$ an $\mathfrak{M}$-primary ideal of $A$. Then the graded local cohomology of $R$ with respect to $R_{+}$can be compared to that of the ideal $R_{+}$in $R$, considered as an $R$-module, as follows:

(1) $H_{R_{+}}^{i}\left(R_{+}\right)_{n} \cong H_{R_{+}}^{i}(R)_{n} \quad$ for all $i \geq 2$ and all $n$. 
(2) $H_{R_{+}}^{0}\left(R_{+}\right)_{n} \cong H_{R_{+}}^{0}(R)_{n} \quad$ for all $n \neq 0$.

(3) $H_{R_{+}}^{1}\left(R_{+}\right)_{n} \cong H_{R_{+}}^{1}(R)_{n} \quad$ for all $n \neq 0$.

(4) There exists an exact sequence of $R$-modules:

$0 \rightarrow H_{R_{+}}^{0}\left(R_{+}\right)_{0} \rightarrow H_{R_{+}}^{0}(R)_{0} \rightarrow{ }_{R} A \rightarrow H_{R_{+}}^{1}\left(R_{+}\right)_{0} \rightarrow H_{R_{+}}^{1}(R)_{0} \rightarrow 0$,

where ${ }_{R} A$ denotes $A$ viewed as an $R$-module $\left(A \cong R / R_{+}\right)$.

(5) If depth $A \geq 1$, then $H_{R_{+}}^{0}\left(R_{+}\right)=H_{R_{+}}^{0}(R)=0$.

Proof. If $x$ is a non-zero-divisor of $A$ then $x t$ is a non-zero-divisor of $R$ in $R_{+}$so that $H_{R_{+}}^{0}(R)=0$. From the exact sequence of $R$-modules

$$
0 \longrightarrow R_{+} \longrightarrow R \longrightarrow{ }_{R} A \longrightarrow 0
$$

one deduces all of the other information by examining the corresponding long exact sequence on local cohomology, noting that $R_{+}$annihilates ${ }_{R} A$, so that $H_{R_{+}}^{0}\left({ }_{R} A\right)={ }_{R} A$, and $H_{R_{+}}^{i}\left({ }_{R} A\right)=0, i>0$.

Lemma 1.2. For all $n \neq-1$, there exists a long exact sequence of the form

$$
\begin{aligned}
0 & \rightarrow H_{R_{+}}^{0}(R)_{n+1} \rightarrow H_{R_{+}}^{0}(R)_{n} \rightarrow H_{R_{+}}^{0}(G)_{n} \rightarrow \\
& \rightarrow H_{R_{+}}^{1}(R)_{n+1} \rightarrow H_{R_{+}}^{1}(R)_{n} \rightarrow H_{R_{+}}^{1}(G)_{n} \rightarrow \cdots \\
\cdots \rightarrow H_{R_{+}}^{d-1}(G)_{n} & \rightarrow H_{R_{+}}^{d}(R)_{n+1} \rightarrow H_{R_{+}}^{d}(R)_{n} \rightarrow H_{R_{+}}^{d}(G)_{n} \rightarrow 0 .
\end{aligned}
$$

Moreover,

$$
\begin{aligned}
H_{R_{+}}^{1}(R)_{n} \rightarrow H_{R_{+}}^{1}(G)_{n} & \rightarrow \cdots \\
& \rightarrow H_{R_{+}}^{d-1}(G)_{n} \rightarrow H_{R_{+}}^{d}(R)_{n+1} \rightarrow H_{R_{+}}^{d}(R)_{n} \rightarrow H_{R_{+}}^{d}(G)_{n} \rightarrow 0
\end{aligned}
$$

is exact for all $n \in \mathbb{Z}$.

Proof. We start with the (graded) exact sequence of $R$-modules:

$$
0 \longrightarrow R_{+}(1) \longrightarrow R \longrightarrow G \longrightarrow 0
$$

This gives rise to the corresponding long (graded) exact sequence on local cohomology, which yields on the $n$th level, for all $n$ :

$$
\begin{aligned}
0 & \rightarrow H_{R_{+}}^{0}\left(R_{+}\right)_{n+1} \rightarrow H_{R_{+}}^{0}(R)_{n} \rightarrow H_{R_{+}}^{0}(G)_{n} \rightarrow \\
& \rightarrow H_{R_{+}}^{1}\left(R_{+}\right)_{n+1} \rightarrow H_{R_{+}}^{1}(R)_{n} \rightarrow H_{R_{+}}^{1}(G)_{n} \rightarrow \cdots \\
\cdots \rightarrow H_{R_{+}}^{d-1}(G)_{n} & \rightarrow H_{R_{+}}^{d}\left(R_{+}\right)_{n+1} \rightarrow H_{R_{+}}^{d}(R)_{n} \rightarrow H_{R_{+}}^{d}(G)_{n} \rightarrow 0 \text { exact. }
\end{aligned}
$$

If $n \neq-1, n+1 \neq 0$, so by Lemma 1.1 parts (2) and (3) we have that for $i=0$ or $1, H_{R_{+}}^{i}\left(R_{+}\right)_{n+1} \cong H_{R_{+}}^{i}(R)_{n+1}$, and this is in fact true for all $n$ if $i>1$ by part (1) of Lemma 1.1. The result follows.

Graded local cohomology is useful for our purposes only when we know that the individual graded pieces have finite length. Note that in our situation $\ell_{A}\left(H_{R_{+}}^{i}(G)_{n}\right)<\infty$ for all $i$ and $n$. This is because, first, by the change of rings principle for local cohomology, and using the exact sequence (3) above, $H_{R_{+}}^{i}(G)=H_{G_{+}}^{i}(G)$ for all $i \geq 0$. As $G_{0}=A / I$ is Artinian, these latter local cohomology modules are well known to be Artinian $G$-modules, and as each of their graded pieces, $H_{G_{+}}^{i}(G)_{n}$ is seen directly to be a finitely-generated $(A / I)$ module, we conclude that all of these have finite length. 
We will come across numerical functions in the sequel. By a numerical function we mean a function $f: \mathbb{Z} \rightarrow \mathbb{Z}$. We introduce the notation $\Delta^{1}(f(n)):=$ $f(n+1)-f(n)$. The following two facts are our main tools for handling numerical functions.

Principle of Algebraic Differentiation. If $f(n)$ and $g(n)$ are both zero for all $n>>0$, and if $\Delta^{1}(f(n))=\Delta^{1}(g(n))$ for all $n \in \mathbb{Z}$ then $f(n)=g(n)$ for all $n \in \mathbb{Z}$.

Proof. $\Delta^{1}(f(n))=\Delta^{1}(g(n))$ for all $n \in \mathbb{Z}$ guarantees that $f(n)=g(n)+$ (a constant) for all $n \in \mathbb{Z}$, and the other hypothesis guarantees that this constant is zero.

Serre's Formula [O, Lemma 2.1]. If $B=B_{0} \oplus B_{1} \oplus B_{2} \oplus \cdots=B_{0}\left[B_{1}\right]$ is a nonnegatively graded homogeneous Noetherian ring over the Artinian local ring $B_{0}$, if $M$ is a $\mathbb{Z}$-graded Noetherian module over $B$, such that $\operatorname{dim}(M)=m$, and if $H^{\prime}(n):=\ell_{B_{0}}\left(M_{n}\right)<\infty$ with $P^{\prime}(n)$ the corresponding Hilbert polynomial, then

$$
\sum_{i=0}^{m}(-1)^{i} \ell_{B_{0}}\left(H_{B_{+}}^{i}(M)_{n}\right)=H^{\prime}(n)-P^{\prime}(n) .
$$

As mentioned by $\mathrm{P}$. Schenzel in [Sz, Theorem C, Beweis], this formula is essentially due to J.-P. Serre, in [Se, No. 80], although he did not use the language of local cohomology. A. Ooishi's paper [O] provides an elementary, direct proof.

Using that the $n$th graded piece of the $i$ th local cohomology module of $G$ with respect to $R_{+}$has finite length, we immediately have by Serre's Formula that if $H_{I}^{\prime}(n)$ represents the Hilbert function of $G, H_{I}^{\prime}(n):=\ell_{A}\left(I^{n} / I^{n+1}\right)$, and if $P_{I}^{\prime}(n)$ represents the corresponding Hilbert polynomial, then for all $n$,

$$
\begin{aligned}
& \sum_{i=0}^{d}(-1)^{i} \ell_{A}\left(H_{R_{+}}^{i}(G)_{n}\right)=H_{I}^{\prime}(n)-P_{I}^{\prime}(n) \\
& \quad=\left[H_{I}(n+1)-H_{I}(n)\right]-\left[P_{I}(n+1)-P_{I}(n)\right]=\Delta^{1}\left(H_{I}(n)-P_{I}(n)\right) .
\end{aligned}
$$

Thus:

Corollary. If $(A, \mathfrak{M}, d)$ is a local ring having an $\mathfrak{M}$-primary ideal $I$, and if $G$ represents the associated graded ring of $A$ with respect to $I$ and $R$ the Rees algebra of $A$ with respect to $I$, then for all $n \in \mathbb{Z}$

$$
\sum_{i=0}^{d}(-1)^{i} \ell_{A}\left(H_{R_{+}}^{i}(G)_{n}\right)=\Delta^{1}\left(H_{I}(n)-P_{I}(n)\right) .
$$

\section{AN APPLICATION OF TWO THEOREMS OF SERRE'S}

In this section we use Serre's well-known theorem on the finiteness of the sheaf cohomology modules for a coherent algebraic sheaf of modules in order to deduce that certain graded pieces of graded local cohomology have finite length, Theorem 2.3 below. As an almost immediate corollary, we get our main result, Theorem 2.4 , by applying Serre's Formula from $\S 1$. We begin with a couple of well-known lemmas, to make the picture a little clearer. 
Lemma 2.1. Let $A$ be a Noetherian ring with an ideal $I$ such that $\operatorname{dim}(A / I)=0$. Let $R$ denote the corresponding Rees algebra, $X:=\operatorname{Proj} R, Y:=\operatorname{Spec} A$, and $f: X \rightarrow Y$ the canonical morphism. Then Supp $\left(R^{i} f_{*}\left(\mathscr{O}_{X}\right)\right) \subseteq \operatorname{Supp}(A / I)$, for all $i \geq 1$.

Proof. We must show that if $P \in \operatorname{Spec}(A) \backslash V(I)$, then $R^{i} f_{*}\left(\mathscr{O}_{X}\right)_{P}=0$. Take an injective resolution of $\mathscr{O}_{X}$ (in the category of $\mathscr{O}_{X}$-modules on $\left.X\right): 0 \rightarrow$ $\mathscr{O}_{X} \rightarrow \mathscr{E}^{0} \rightarrow \mathscr{E}^{1} \rightarrow \cdots$. Then $R^{i} f_{*}\left(\mathscr{O}_{X}\right)_{P}$ is the cohomology of $: \cdots \rightarrow$ $f_{*}\left(\mathscr{E}^{i-1}\right)_{P} \rightarrow f_{*}\left(\mathscr{E}^{i}\right)_{P} \rightarrow f_{*}\left(\mathscr{E}^{i+1}\right)_{P} \rightarrow \cdots$. But $f$ is an isomorphism outside $V(I)$ by [Ha, II.7.13(b)]. Hence the cohomology in question is equal to that of: $\cdots \rightarrow\left(\mathscr{E}^{i-1}\right)_{Q} \rightarrow\left(\mathscr{E}^{i}\right)_{Q} \rightarrow\left(\mathscr{E}^{i+1}\right)_{Q} \rightarrow \cdots$, where $Q:=f^{-1}(P)$. But this cohomology is 0 , as $\cdots \rightarrow \mathscr{E}^{i-1} \rightarrow \mathscr{E}^{i} \rightarrow \mathscr{E}^{i-1} \rightarrow \cdots \quad$ is exact (at all stalks).

Lemma 2.2. Let $R$ be any Noetherian ring having ideal $I \neq 0$, and let $M$ be a finite $R$-module. Let $\bar{M}:=M / \mathrm{H}_{I}^{0}(M)$. Then for all $i>0, H_{I}^{i}(M) \cong H_{I}^{i}(\bar{M})$. Proof. By looking at the Čech complex $C$. which is the direct limit of Koszul complexes, one can see that if $N$ is an $R$-module all of whose elements are annihilated by a power of $I$, then $H_{I}^{i}(N)=0$ for all $i>0$. In particular, we see that $H_{I}^{i}\left(H_{I}^{0}(M)\right)=0$ for all $i>0$. The result now follows by considering the long exact sequence on local cohomology associated to the short exact sequence: $0 \longrightarrow H_{I}^{0}(M) \longrightarrow M \longrightarrow 0$.

The next theorem is the essence of this paper, showing that each $n$th graded piece of the $i$ th local cohomology modules $0 \leq i \leq d$ of the Rees algebra with respect to the positively graded ideal has finite length when $n$ is nonnegative. We note that Sally, using ideal-theoretic calculations, proved a similar result for $i=d$, in the case that $I=\mathfrak{M}$, the maximal ideal ([Sa, Bottom of p. 233]), but her result holds in fact for $\mathfrak{M}$-primary $I$.

Theorem 2.3. Let $(A, \mathfrak{M})$ be a local ring, and let $I$ be any $\mathfrak{M}$-primary ideal of $A$. Then $\ell_{A}\left(H_{R_{+}}^{i}(R)_{n}\right)<\infty$ for all $n \geq 0$, for all $i \geq 0$.

Proof. We will handle the case $i=0$ separately. As $H_{R_{+}}^{0}(R)_{n}=\left\{a \in I^{n} \mid a I^{j}=\right.$ 0 for all $j>>0\}=I^{n} \cap \bigcup_{j}\left(0: I^{j}\right)$, it suffices to show that $\bigcup_{j}\left(0: I^{j}\right)$ has finite length as an $A$-module. This module is finitely generated, so we merely need to see that it has support solely in the maximal ideal. But for some $m>>0, I^{m} \subseteq$ Ann $\left(\bigcup_{j}\left(0: I^{j}\right)\right)$.

Now assume $i \geq 1$. By applying Lemma 2.2, we may assume without loss of generality that $\bar{H}_{R_{+}}^{0}(R)=0$. We induct on $n$, using the long exact sequence on local cohomology from Lemma 1.2. For $n \geq 0, H_{R_{+}}^{i-1}(G)_{n}$ has finite length (as an $A$-module) and we have $H_{R_{+}}^{i}\left(R_{+}\right)_{n+1} \cong H_{R_{+}}^{i}(R)_{n+1}$, hence $H_{R_{+}}^{i}(R)_{n+1}$ must have finite length, as soon as $H_{R_{+}}^{i}(R)_{n}$ does. Thus the problem has been reduced to showing that $\ell_{A}\left(H_{R_{+}}^{i}(R)_{0}\right)<\infty$ for $i \geq 1$.

For this, we use the two exact sequences, (1) and (2), which connect local and global cohomology.

By assumption, $H_{R_{+}}^{0}(R)=0$, so on the zeroeth level, to show that $H_{R_{+}}^{1}(R)_{0}$ has finite length, it suffices to show that $H^{0}\left(X, \mathscr{O}_{X}\right) / A$ has finite length, using (1). As $H^{0}\left(X, \mathscr{O}_{X}\right)=\bigcap_{i} A\left[\frac{I}{a_{i}}\right]$, where $I=\left(a_{1}, \ldots, a_{n}\right), H^{0}\left(X, \mathscr{O}_{X}\right) / A$ is an $(A / I)$-module, hence has finite length as an $A$-module if and only if $H^{0}\left(X, \mathscr{O}_{X}\right)$ 
is finitely generated as an $A$-module. Set $Y:=\operatorname{Spec} A$. Then $X$ is a projective scheme over $Y$ under the canonical morphism, so by Serre's Theorem [Ha, Theorem III.5.2(a)], $H^{i}\left(X, \mathscr{O}_{X}\right)$ is a finitely generated $A$-module for all $i \geq 0$.

Using (2), it remains to show that $H^{i}\left(X, \mathscr{O}_{X}\right)$ has finite length for $i \geq 1$. We have by [Ha, Corollary III.8.6] that $R^{i} f_{*}\left(\mathscr{O}_{X}\right) \cong H^{i}\left(X, \mathscr{O}_{X}\right)^{\sim}$ (sheafification over $Y)$, where $H^{i}\left(X, \mathscr{O}_{X}\right)$ is considered as an $A$-module. Hence the finitely generated $A$-module $H^{i}\left(X, \mathscr{O}_{X}\right)$ has finite length if and only if $R^{i} f_{*}\left(\mathscr{O}_{X}\right)$ is supported in only finitely many maximal ideals. But by Lemma 2.1, $\operatorname{Supp}\left(R^{i} f_{*}\left(\mathscr{O}_{X}\right)\right) \subseteq \operatorname{Supp}(A / I)$, a finite set of maximal ideals.

We now have all of the ingredients to easily obtain our main result.

Theorem 2.4. If $(A, \mathfrak{M}, d)$ is a local ring and if $I$ is an $\mathfrak{M}$-primary ideal of $A$ then for all $n \geq 0$,

$$
\sum_{i=0}^{d}(-1)^{i} \ell_{A}\left(H_{R_{+}}^{i}(R)_{n}\right)=(-1)^{d}\left[P_{I}(n)-H_{I}(n)\right] .
$$

Proof. Let $\phi(n)$ denote the numerical function defined by

$$
\phi(n):=\sum_{i=0}^{d}(-1)^{i} \ell_{A}\left(H_{R_{+}}^{i}(R)_{n}\right) .
$$

From the exact sequence of Lemma 1.2, we immediately conclude that

$$
\begin{aligned}
\sum_{i=0}^{d}(-1)^{i} \ell_{A}\left(H_{R_{+}}^{i}(G)_{n}\right) & =\sum_{i=0}^{d}(-1)^{i} \ell_{A}\left(H_{R_{+}}^{i}(R)_{n}\right)-\sum_{i=0}^{d}(-1)^{i} \ell_{A}\left(H_{R_{+}}^{i}(R)_{n+1}\right) \\
& =-\Delta^{1}(\phi(n)) .
\end{aligned}
$$

On the other hand, from the Corollary to Serre's Formula, we have that

$$
\sum_{i=0}^{d}(-1)^{i} \ell_{A}\left(H_{R_{+}}^{i}(G)_{n}\right)=(-1)^{d} \Delta^{1}\left(H_{I}(n)-P_{I}(n)\right),
$$

and the result follows by the Principle of Algebraic Differentiation.

\section{SPECialized Results}

Our purpose in this final section is to use some additional hypotheses to draw stronger conclusions than Theorem 2.4. In particular, we will show how to recover Sally's results mentioned in the introduction. First we note that by making a strong hypothesis on the associated graded ring of $A$ with respect to $I, G$, one can draw a stronger conclusion. We provide an example to show that this conclusion fails without this stronger hypothesis.

Proposition 3.1. If $(A, \mathfrak{M}, d)$ is a local ring with $d \neq 1$, and if $I$ is an $\mathfrak{M}$ primary ideal of $A$ such that the associated graded ring, $G$, of $A$ with respect to $I$ is Cohen-Macaulay, then

$$
\ell_{A}\left(H_{R_{+}}^{d}(R)_{n}\right)=(-1)^{d}\left[P_{I}(n)-H_{I}(n)\right]
$$

for all $n \in \mathbb{Z}$. The same result holds in case $d=1$ for all $n \neq-1$.

Proof. The assumption that $G$ is Cohen-Macaulay can be viewed as the statement that $H_{M}^{i}(G)=0$ for all $i \leq d-1$. The Artinianness of $A / I$ implies that 
$M=\sqrt{G_{+}}$so that $H_{M}^{d-1}(G)=H_{G_{+}}^{d-1}(G)$; and $H_{G_{+}}^{d-1}(G)=H_{R_{+}}^{d-1}(G)$ by the change of rings principle for local cohomology. Hence we may rewrite the long exact sequence of Lemma 1.2 as

$$
0 \longrightarrow H_{R_{+}}^{d}(R)_{n+1} \longrightarrow H_{R_{+}}^{d}(R)_{n} \longrightarrow H_{R_{+}}^{d}(G)_{n} \longrightarrow 0 .
$$

This allows us to conclude, if $d \neq 1$, that for all $n \in \mathbb{Z}$ (and if $d=1$, then for all $n \neq-1$ )

$$
\ell_{A}\left(H_{R_{+}}^{d}(G)_{n}\right)=\ell_{A}\left(H_{R_{+}}^{d}(R)_{n}\right)-\ell_{A}\left(H_{R_{+}}^{d}(R)_{n+1}\right)=-\Delta^{1}\left(\ell_{A}\left(H_{R_{+}}^{d}(R)_{n}\right)\right) .
$$

The result now follows by the Principle of Algebraic Differentiation exactly as in the proof of Theorem 2.4.

Example. Proposition 3.1 is false if $G$ is not Cohen-Macaulay. To see this we utilize an example constructed by $\mathrm{S}$. Huckaba in a different context $[\mathrm{Hb}$, Example 3.1]. Let $A:=k[[X, Y]]$, where $k$ is any field and $X$ and $Y$ are indeterminates, and let $I:=\left(X^{7}, X^{6} Y, X^{2} Y^{5}, Y^{7}\right)$. Then for $n>4$, the Hilbert function $H_{I}(n):=\ell_{A}\left(A / I^{n}\right)$ is equal to the corresponding Hilbert polynomial, $P_{I}(n)=49\left(\begin{array}{c}n+1 \\ 2\end{array}\right)-21\left(\begin{array}{l}n \\ 1\end{array}\right)+3$.

However, we can directly compute that

$$
\begin{aligned}
& H_{I}(1)=32, P_{I}(1)=31 ; \quad H_{I}(2)=110, P_{I}(2)=108 ; \\
& H_{I}(3)=235, P_{I}(3)=234 \text {, }
\end{aligned}
$$

so that $P_{I}(n)-H_{I}(n)<0$ for $n=1,2,3$. For the proposition to hold, $P_{I}(n)-$ $H_{I}(n)$ would have to be nonnegative for all $n$.

What remains unclear is the extent to which one can recover some sort of reasonable result for $n<0$, without the strong hypothesis on $G$. Work by Sally [Sa, Proposition 3], Huckaba [Hb, Theorem 2.1], and T. Marley [Ma] indicates that many nice theorems which are true for $\mathfrak{M}$-primary ideals as in the proposition remain true for $m$-primary ideals whose associated graded rings have depth $d-1$. However, even in the first interesting case, that of a 2dimensional Cohen-Macaulay local ring $A$, one runs into the problem that the negative pieces of the local cohomology modules need no longer have finite length. For example, Sally showed [Sa, Proposition 1 (iii)] that $H_{R_{+}}^{1}(R)_{-1} \cong A$.

A different approach to negative pieces can also be taken, following Kirby and Mehran, by replacing the usual Rees algebra with one which is extended to finitely many negative places (cf. [K-M, bottom of page 459]). One checks that the arguments in the previous section easily go through to this "generalized" Rees algebra. However, this creates a different set of negatively-graded pieces, leaving open the question of the nature of the naturally occurring negative pieces.

We ask in particular:

Question 3.2. Does some version of Proposition 3.1 continue to hold under the weaker hypothesis that depth $G \geq d-1$ ?

We conclude with a demonstration of how a strengthened version of Sally's result mentioned in the introduction can be obtained from Theorem 2.4. 
Proposition 3.3. Let $(A, \mathfrak{M}, 2)$ be Cohen-Macaulay and let $I$ be any $\mathfrak{M}$ primary ideal of $A$. If $R$ is the Rees algebra of $A$ with respect to $I$, then

$$
\begin{aligned}
\ell_{A}\left(H_{R_{+}}^{2}(R)_{0}\right) & =e_{2}, \\
\ell_{A}\left(H_{R_{+}}^{2}(R)_{1}\right) & =e_{0}-e_{1}+e_{2}-\ell(A / \widetilde{I}), \\
\ell_{A}\left(H_{R_{+}}^{2}(R)_{-1}\right) & =e_{1}+e_{2},
\end{aligned}
$$

where $\tilde{I}$ represents the Ratliff-Rush ideal associated to $I$.

Proof. If $x \in A$ is a non-zero-divisor then $x t \in R_{1}$ is as well, so $H_{R_{+}}^{0}\left(R_{+}\right)=0$. Also, $A$ satisfying Serre's condition $\mathscr{S}_{2}$ implies that $A$ is its own $\mathscr{S}_{2}$-ification and hence that $H_{R_{+}}^{1}(R)=0$ (cf. [Sa, Remark 2]). Similarly, one sees that $H_{R_{+}}^{1}(R)_{1} \cong \tilde{I} / I$. Both of these statements follow from the exact sequence (1). (See [HJLS2] for more information about Ratliff-Rush ideals.) Plugging 0 in for $n$ in Theorem 2.4 then yields statement (i). Plugging 1 in Theorem 2.4 yields $\ell\left(H_{R_{+}}^{2}(R)_{1}\right)-\ell(\widetilde{I} / I)=P_{I}(1)-H_{I}(1)=e_{0}-e_{1}+e_{2}-\ell(A / I)$, and hence statement (ii). Statement (iii) requires a more careful analysis.

First look closely at the long exact sequence from the proof of Lemma 1.2 in the case $n=-1$. Noticing that, as $G$ and hence $H_{R_{+}}^{0}(G)$ are nonzero only in positive degrees, and applying part (1) of Lemma 1.1, we see that this reduces to

$$
\begin{aligned}
0 \rightarrow H_{R_{+}}^{1}\left(R_{+}\right)_{0} & \rightarrow H_{R_{+}}^{1}(R)_{-1} \rightarrow H_{R_{+}}^{1}(G)_{-1} \rightarrow \\
H_{R_{+}}^{2}(R)_{0} & \rightarrow H_{R_{+}}^{2}(R)_{-1} \rightarrow H_{R_{+}}^{2}(G)_{-1} \rightarrow 0 .
\end{aligned}
$$

Let $f$ denote the map $H_{R_{+}}^{1}(R)_{-1} \rightarrow H_{R_{+}}^{1}(G)_{-1}$ in the sequence above. We claim that $f$ is zero. Notice first that $H_{R_{+}}^{1}(R)_{-1} \cong A$ as an $A$-module (cf. [Sa, Proposition 2 (iii)] -this proof requires that $A$ be Cohen-Macaulay). Then by part (5) of Lemma 1.1 we have

$$
0 \longrightarrow A \longrightarrow H_{R_{+}}^{1}\left(R_{+}\right)_{0} \longrightarrow H_{R_{+}}^{1}(R)_{0}=0 \quad \text { exact. }
$$

Thus the first row of the long exact sequence is nothing but

$$
0 \longrightarrow A \stackrel{g}{\longrightarrow} A \stackrel{f}{\longrightarrow} H_{R_{+}}^{1}(G)_{-1} \text {. }
$$

Now $A$ must inject into $A$ as a cyclic module, i.e., a principal ideal. If $g(A) \neq A$, we could conclude that ht $A / g(A) \leq 1$, so $A / g(A)$ would have infinite length and yet be contained in $H_{R_{+}}^{1}(G)_{-1}$, which has finite length, a contradiction. Hence we must have $g(A)=A$ and so $f=0$.

Thence we are left with the exact sequence:

$$
0 \rightarrow H_{R_{+}}^{1}(G)_{-1} \rightarrow H_{R_{+}}^{2}(R)_{0} \rightarrow H_{R_{+}}^{2}(R)_{-1} \rightarrow H_{R_{+}}^{2}(G)_{-1} \rightarrow 0 .
$$

By comparing lengths, using statement (i), and applying the Corollary to Serre's Formula, we conclude that $\ell\left(H_{R_{+}}^{2}(R)_{-1}\right)=e_{2}-\Delta^{1}\left(H_{I}(-1)-P_{I}(-1)\right)=e_{2}-$ $P_{I}(0)+P_{I}(-1)=e_{2}-e_{2}+e_{1}+e_{2}$, as required.

\section{REFERENCES}

[G] S. Goto, Buchsbaum rings with multiplicity 2, J. Algebra 74 (1982), 494-508.

[Ha] R. Hartshorne, Algebraic geometry, Springer-Verlag, Berlin, 1967. 
[HJLS1] W. Heinzer, B. Johnston, D. Lantz, and K. Shah, Coefficient ideals in and blowups of a commutative noetherian domain, J. Algebra 162 (1993), 355-391.

[HJLS2] _ The Ratliff-Rush ideals in a Noetherian ring. A survey, Methods in Module Theory, Marcel Dekker, New York, 1992, pp. 149-159.

[HJL] W. Heinzer, B. Johnston, and D. Lantz, First coefficient domains and ideals of reduction number one, Comm. Algebra 21 (1993), 3797-3827.

[HIO] M. Herrmann, S. Ikeda, and U. Orbanz, Equimultiplicity and blowing-up, Springer-Verlag, Berlin, 1988.

[Hb] S. Huckaba, Reduction numbers for ideals of higher analytic spread, Math. Proc. Cambridge Philos. Soc. 102 (1987), 49-57.

[Hn] C. Huneke, Hilbert functions and symbolic powers, Michigan Math. J. 34 (1987), 293-318.

[K-M] D. Kirby and H. A. Mehran, Hilbert functions and the Koszul complex, J. London Math. Soc. (2) 24 (1981), 459-466.

[Ma] T. Marley, The coefficients of the Hilbert polynomial and the reduction number of an ideal, J. London Math. Soc. (2) 40 (1989), 1-8.

[Mo] M. Morales, Polynôme d'Hilbert-Samuel des clôtures intégrales des puissances d'un idéal m-primaire, Bull. Soc. Math. France 112 (1984), 343-358.

[O] A. Ooishi, Genera and arithmetic genera of commutative rings, Hiroshima Math. J. 17 (1987), 47-66.

[Sa] J. D. Sally, Reductions, local cohomology and Hilbert functions of local rings, Commutative Algebra: Durham 1981 (R.Y. Sharp, ed.), Cambridge Univ. Press, Cambridge, 1982, pp. 231-241.

[Sz] P. Schenzel, Über die freien Auflösungen extremaler Cohen-Macaulay-Ringe, J. Algebra 64 (1980), 93-101.

[Se] J.-P. Serre, Faisceaux algébriques cohérents, Ann. of Math. (2) 61 (1955), 197-278.

(B. Johnston and J. Verma) Department of Mathematics, Louisiana State University, BATON ROUGE, LOUISIANA 70803

(B. Johnston) Department of Mathematics, Florida Atlantic University, Boca Raton, FLORIDA 33431

E-mail address: johnstonøcse.fau.edu

(J. Verma) Indian Institute of Technology, Powai, Bombay, Maharashtra, 400076 India E-mail address: majkvia@cc.iitb.ernet.in 